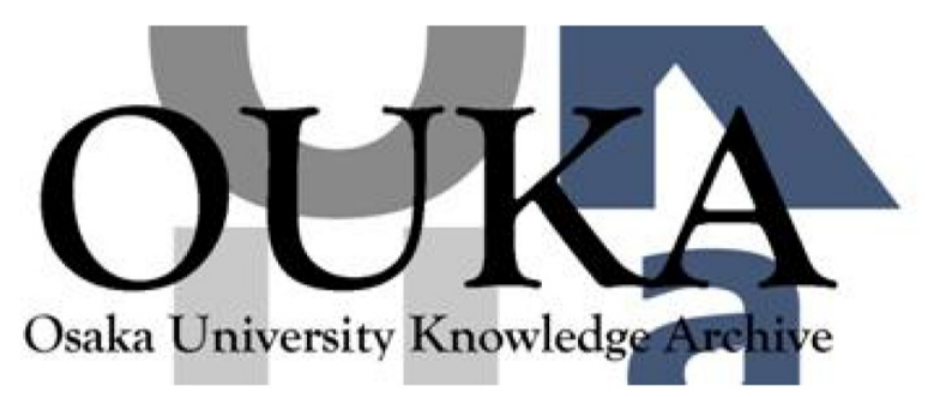

\begin{tabular}{|c|l|}
\hline Title & $\begin{array}{l}\text { Two-photon absorption spectra of luminescent } \\
\text { conducting polymers measured over wide spectral } \\
\text { range }\end{array}$ \\
\hline Author(s) & $\begin{array}{l}\text { Meyer, Ron K.; Liess, Martin; Benner, Robert E. } \\
\text { et al. }\end{array}$ \\
\hline Citation & $\begin{array}{l}\text { Proceedings of SPIE - The International Society } \\
\text { for Optical Eng ineering. 3145 p.219-p. 228 }\end{array}$ \\
\hline Issue Date & $1997-12-01$ \\
\hline oaire:version & VoR \\
\hline URL & https://hdl. handle.net/11094/76955 \\
\hline rights & \\
\hline Note & \\
\hline
\end{tabular}

Osaka University Knowledge Archive : OUKA

https://ir. Library. osaka-u. ac. jp/

Osaka University 


\title{
TWO-PHOTON ABSORPTION SPECTRA OF LUMINESCENT CONDUCTING POLYMERS MEASURED OVER WIDE SPECTRAL RANGE
}

\author{
R. K. Meyer ${ }^{\dagger}$, M. Liess, R. E. Benner ${ }^{\dagger}$, and W. Gellermann, Z. V. Vardeny \\ Departments Electrical Engineering ${ }^{\dagger}$ and Physics, \\ University of Utah, Salt Lake City, Utah 84112 \\ M. Ozaki and K. Yoshino, \\ Depart. of Electronic, Information Systems and Energy Engineering \\ Osaka University, 2-1 Yamada-Oka, Suita, Osaka 565, JAPAN \\ Y. Ding and T. Barton, \\ Department of Chemistry, lowa State University, Ames, lowa 50011
}

\begin{abstract}
We report the two-photon absorption (TPA) spectra of poly(2,5-dibutoxy-p-phenylene acetylene) (PPADBO), poly(2,5-dioctyloxy-p-phenylene vinylene) (PPV-DOO), and poly(3-hexylthiophene) (PAT6) in the spectral range extending from $576 \mathrm{~nm}$ to $846 \mathrm{~nm}$. Using the Z-scan technique on the polymers in solution. we measured a strong two-photon allowed transition in all three materials which we attribute to the $\mathrm{mAg}$ essential state. In the case of PPA-DBO and PPV-DOO, TPA peaks were coincident with dispersion in the nonlinear refractive indices as detected by reduced aperture $Z$ scan. In all three polymers this peak occurs at approximately 1.3 the bandgap energy $\left(E_{g a p}\right)$. The excitonic nature of the excited electronic states in - PPA-DBO is indicated by the lack of a TPA band at or near the $1 \mathrm{~B}_{u}$ exciton position. Saturation was observed in the nonlinear index of refraction near spectral peaks, as well as an apparent reverse KramersKronig effect.
\end{abstract}

Keywords: two-photon absorption, two-photon saturation, luminescent polymers, dispersion, polymer solution.

\section{Introduction}

There is considerable debate concerning the proper (most predictive) model for conducting polymers--excitonic ${ }^{1,2}$ or single particle (band theory). ${ }^{3}$ Most experimental results can be explained with either model if the correct parameters are picked. Both views state that the first allowed state will be the dipole allowed $1 \mathrm{~B}_{u}$ exciton, characterized by a strong and broadened spectral feature in the linear absorption spectrum, and that strong two-photon allowed transition will then be observed above the 1Bu energy. 4-6 Identification of the most appropriate model is crucial to understanding the physical mechanisms involved in the observed luminescent and nonlinear optical properties of these potentially useful polymers. Such an understanding will also provide a predictive tool for the future synthesis of polymers with enhanced optical properties.

Various experiments that probe the two-photon allowed $A_{g}$ states have shown that, in luminescent conducting polymers, a single, strong two-photon allowed transition is observed a few electron volts above the $1 B_{u}$ exciton. ${ }^{7-12}$ It has, therefore, been difficult to attribute the observed $\mathrm{mA}_{g}$ state to one model or the other over the limited spectral range and resolution of the characteristic nonlinear absorption experiment. Only the recent investigation by Lawrence et al. has shown that other two-photon allowed transitions in 
addition to the $\mathrm{mA}_{\mathrm{g}}$ exist above the $1 \mathrm{~B}_{u}$ exciton, which tends to support the excitonic model. ${ }^{13}$ Our experiments and results further support the excitonic model.

In this research three luminescent conducting polymers, namely, poly (paraphenylene vinylene) (PPV), polythiophene (PT), and poly (paraphenylene acetylene) (PPA) are investigated. Each polymer exhibits the typical $\pi$-conjugated backbone with associated alternating bond lengths, as illustrated in the insets of Figs. 1a, 2a, and $3 a$. By measuring the two-photon absorption spectra of PPA-DBO, PPV-DOO, and PAT6 over a wide spectral range, it is possible to clarify and enhance the understanding of the physical mechanisms involved in the observed properties of these potentially useful materials. Employing the Z-scan technique enables accurate identification of actual absorption peaks, since it measures real as well as imaginary $\chi^{(3)}$. This fortuitous feature allows the detection of dispersion in the real part of $\chi^{(3)}$, which must accompany two-photon absorption. Furthermore, Z scan, since it can directly detect the signs of the complex $\chi^{(3)}$ components, avoids the misidentification of one-photon saturation effects as two-photon spectral peaks. 5,14

To eliminate anomalous effects associated with optical damage and surface inhomogeneities, samples were prepared in solution. Therefore, the values obtained for the nonlinear index of refraction (real part), $n_{2}$ and two-photon absorption, $\alpha_{2}$ will not reflect the actual $\chi^{(3)}$ of the condensed material; yet, the relative intensities and position of the absorption peaks will remain unchanged. To verify this, linear absorption spectra (Figs. 1-3) were obtained for each polymer solution using a Perkin Elmer Lambda 9 spectrophotometer, and compared to the linear absorption of thin film samples. The solid state and liquid linear absorptions were, essentially, identical.

Of particular note in all the linear absorption spectra is the characteristically broad feature at the $1 \mathrm{~B}_{u}$ exciton and the small additional feature at the higher energies $(3.5-4.0 \mathrm{eV})$. We attribute this additional feature to the $\mathrm{nB}_{\mathrm{u}}$ state which may indicate the beginning of the free-electron continuum. ${ }^{15}$ Since this feature occurs at approximately the same position in different materials, it suggests that the peak arises from the $\pi$-conjugation common to all three materials. The absorption spectra begin to rise precipitously at $4.5 \mathrm{eV}$ in both PPA-DBO and PPV-DOO, which is indicative of the first excited state of the individual constituent benzene rings. ${ }^{16-18}$ Careful inspection of the spectra further reveals that PPA has the sharpest, least broadened peaks.

\section{Experiment}

To obtain the high peak powers required for Z-scan experiments, a regeneratively-pumped dye amplifier system has been employed. A Quantronix frequency-doubled Nd:YAG laser provides excitation for a synchronously pumped dye laser. A three-stage dye amplifier was built to amplify the $76 \mathrm{MHz}$ dye laser pulses, thereby generating high peak powers at the desired wavelengths. The high repetition rate dye laser pulses are amplified up to 50,000 times, resulting in $10 \mathrm{psec}, 250 \mathrm{~Hz}$ to $2 \mathrm{kHz}$ pulses at up to 300 $\mathrm{GW} / \mathrm{cm}^{2}$ peak intensity. Using available dyes, a tuning range from $576 \mathrm{~nm}$ to $846 \mathrm{~nm}$ has been demonstrated. The wavelength regime between $770 \mathrm{~nm}$ and $790 \mathrm{~nm}$ was obtained by employing a regeneratively amplified Ti:sapphire laser system.

Solutions of PPV-DOO and PAT6 were prepared by dissolving small amounts of thin films of these materials in chloroform solvent. PPA-DBO was prepared by dissolving its powder form in chloroform solvent. The solutions were allowed to stabilize for three days to ensure that the solutes had completely dissolved. This was verified by regular linear absorption spectra taken over the three-day period. Determining the optimum concentration involved a trial and error procedure. The solution concentration was increased until a good Z-scan signal could be achieved at peak powers at least half that required for a measurable $\mathrm{CS}_{2}$ signal. These optimization $Z$ scans were conducted at $825 \mathrm{~nm}(2 \hbar \omega=3.0 \mathrm{eV})$, in a regime where strong two-photon absorption is not expected. Once the optimum concentration had been achieved, linear absorption was measured at an appropriate wavelength. Strong linear absorption at the absorption maximum exceeded the capability of the measuring equipment. Therefore, the measurements were conducted at $540 \mathrm{~nm}$, further from the onset of strong absorption.

Open and reduced-aperture $Z$ scans were conducted on all three polymers simultaneously. $C_{2}$ was used to determine peak on-axis intensities at each wavelength, since it has good optical quality, a large, 
fast, dispersionless $n_{2}$, and has been previously well-characterized by absolute Z-scan measurements, where measured power was used directly to calculate $n_{2}$.

We employed a comparative Z-scan procedure, wherein, we perform a reduced-aperture $Z$ scan on $\mathrm{CS}_{2}$ immediately followed by reduced and open-aperture $Z$ scans on each polymer solution at a particular wavelength. A representative group of $Z$ scans for a particular wavelength consists of one $C S_{2}$ reducedaperture scan and six reduced and open-aperture scans of a polymer at various on-axis intensities. The peak on-axis intensity, $I_{0}$ is then calculated from the $\mathrm{CS}_{2}$ peak to valley transmission change using previously measured values for its nonlinear index of refraction. Based on the seminal work of Sheik-Bahae et $a l$., the equation for $l_{0}$ is given by ${ }^{19}$

$$
\mathrm{I}_{0}=\frac{\Delta \mathrm{T}_{\mathrm{PV}} \lambda(10)^{-\mathrm{OD}}}{5.6 \times 10^{-15}}\left(\mathrm{~W} / \mathrm{cm}^{2}\right)
$$

where, $\Delta T_{P V}$ is the maximum transmission change observed in the reduced aperture $C_{2}$ scan, and $O D$ refers to the total optical density of the neutral density filters. This equation takes into consideration losses as a result of reflections from the glass cuvette.

This procedure was implemented to alleviate the problem of determining average power of the amplified pulses at wavelengths where the dye amplifier was amplifying pulses less than 10,000 times. At these positions the average power from the $76 \mathrm{MHz}$ background exceeded the average power of the $1 \mathrm{kHz}$ amplified pulse, thereby making it impossible to distinguish between the two. $\mathrm{CS}_{2}$ scans remedied this problem, since the positive optical Kerr effect is only sensitive to high peak powers, is relatively dispersionless, and can be used to indirectly measure the peak power from the dye amplifier using Eq. (1).

Z-scan measurements were conducted over a spectral range of $576 \mathrm{~nm}$ to $846 \mathrm{~nm}$ using various laser dyes in the three-stage dye amplifier system. A pulse repetition rate of $1 \mathrm{kHz}$ and lock-in detection was used to reduce noise. Z-scan data were saved and analyzed graphically to acquire the transmission changes. The nonlinear index of refraction, $n_{2}$, was then directly calculated from the transmission change in the manner discussed in Ref. 19. The nonlinear absorption, $\alpha_{2}$, was calculated at the $z=0$ position (point of highest intensity) by iteratively solving the equation

$$
\frac{\mathrm{dl}(\mathrm{r})}{\mathrm{dz}}=-\alpha_{0} \mathrm{I}-\alpha_{2} \mathrm{I}^{2}
$$

where $I(r)$ is the gaussian distributed intensity and $\alpha_{0}$ is the linear absorption.

\section{Results}

Real and imaginary molecular second hyperpolarizabilities, respectively, are plotted in Figs. 1-3 parts (b) and (c) for PPV-DOO, PPA-DBO, and PAT6. Imaginary molecular second hyperpolarizability, $\gamma "$ ", corresponds to two-photon absorption, $\alpha_{2}$ within a multiplicative factor, while real molecular second hyperpolarizability, $\gamma^{\prime}$, corresponds to nonlinear dispersion, $n_{2}$. A guide for the eye is included to accentuate features suggested by the data points. The $\gamma^{\prime}$ spectra for all polymers clearly indicates dispersion near $\gamma^{\prime \prime}$ peaks, as expected from Kramers-Kronig analysis.

In all three polymers a strong peak in two-photon absorption $\left(\mathrm{mA}_{\mathrm{g}}\right)$ is evident at 0.5 to $0.6 \mathrm{eV}$ above the first one-photon exciton $\left(1 \mathrm{~B}_{\mathrm{u}}\right)$. In addition to strong $\mathrm{mA}_{\mathrm{g}}$ absorption peaks, there appear to be additional smaller peaks in all three polymers. Although the $\alpha_{2}$ data for the secondary peaks are not as convincing as that for the $\mathrm{mA}_{g}$, the dispersion in $n_{2}$ near these points confirms their existence. It appears that for all three polymers, an additional $A_{\theta}$ character state exists approximately $0.3 \mathrm{eV}$ above the essential $\mathrm{mA}_{g}$ state. In both PPA-DBO and PAT6, there is evidence of a third $A_{g}$ state coincident or slightly above a secondary one-photon allowed spectral peak seen in the linear absorption data (Figs. 1-3). These peaks are also confirmed by the dispersion seen in $n_{2}$. The peak occurs at approximately $3.55 \mathrm{eV}$ in PAT6 and $4.0 \mathrm{eV}$ in PPA-DBO. 


\section{Discussion and Analysis}

Our results for the position of the $\mathrm{mA}_{\mathrm{g}}$ state in each polymer is in good agreement with the results of others using a variety of experimental techniques. Jeglinski et al. have shown, through electroabsorption experiments conducted on PPA thin films, that there is a possible $A_{q}$ state $0.6 \mathrm{eV}$ above the $1 \mathrm{~B}_{u}$ exciton at $2.6 \mathrm{eV} .9,11$ This is a larger separation between the $1 \mathrm{~B}_{u}$ and $\mathrm{mA}_{\mathrm{g}}$ than seen in PPV-DOO or PAT6, and may be attributed to more dimerization as a result of the triple carbon-carbon bonds. A similar large separation between the $1 B_{u}$ and $m A_{g}$ state is seen in polysilanes, where the separation is as great as $1.0 \mathrm{eV}$ and the bond length alternation parameter is significantly greater than that in PPA-DBO, PPV-DOO, or PAT6. 20, 21 Lemmer et al. and Baker et al. have independently conducted two-photon fluorescence measurements on PPV.7, 10 Their results clearly indicate an intense $A_{g}$ character state approximately 0.5 $\mathrm{eV}$ above the $1 \mathrm{~B}_{u}$ exciton. Third-harmonic generation (THG) experimental results also show the $1 \mathrm{~B}_{u}$ and $a$ single strong $A_{g}$ character state at similar spectral positions. ${ }^{22}$ Although we were unable to confirm the position of our observed strong two-photon absorption peak $\left(\mathrm{mA}_{g}\right)$ in PAT6 with coincident dispersion in $n_{2}$, Pfeffer et al. ${ }^{12}$ and $\mathrm{Cha}$ et al. ${ }^{8}$ have separately identified the peak near the same position $(0.5$ to $0.6 \mathrm{eV}$ above the $1 B_{u}$ exciton). Pfeffer et al. have used the Kerr ellipsometry technique on polythiophene solutions to identify a pair of two-photon absorption peaks; one at $0.6 \mathrm{eV}$ above the $1 \mathrm{~B}_{\mathrm{u}}$ exciton, and another small peak on the low energy side of the $1 \mathrm{~B}_{u}$ transition. ${ }^{12}$ Cha et al. have measured the twophoton spectra of polythiophene thin films by using a combination of third-harmonic generation and nondegenerate four-wave mixing. Their results indicate a two-photon peak at approximately $0.5 \mathrm{eV}$ above the $1 \mathrm{~B}_{u}$ exciton. ${ }^{8}$ They also attempted to fit their results using a four-essential states model $\left(1 \mathrm{~A}_{g}, 1 \mathrm{~B}_{u}, \mathrm{~mA}_{\mathrm{g}}\right.$ $\left.n B_{u}\right) .15$ Thus, there is good agreement in all three polymers with previously reported results in regard to the position of the $\mathrm{mA}_{\mathrm{g}}$ peaks.

A comparative analysis of the two-photon spectra indicates that PPA-DBO has sharper, more welldefined two-photon features than PPV-DOO or PAT6. This characteristic was also observed in its linear absorption spectrum (Fig. 2). As suggested by Soos et al., this can be attributed to more dimerization, which results in further separation between $1 \mathrm{~B}_{u}$ and $\mathrm{mA}_{g}$ spectral signatures and less electron-phonon coupling. ${ }^{21}$ Alternatively, the sharper spectral features can be attributed to less disorder. ${ }^{23}$ This can occur also as a result of more dimerization and less delocalization, which limits disorder inducing defects.

By plotting linear and nonlinear absorption together for all three materials in Fig. 4 (a-c), the excitonic character of the observed spectra is self-evident. The first observed $A_{g}$ state $\left(m A_{g}\right)$ is not coincident with the $1 B_{u}$ exciton. Moreover, the higher energy $A_{g}$ states begin to coincide with the higher energy $B_{u}$ state observed in the linear spectra (Figs. 1a-3a), thereby suggesting the onset of the freeelectron continuum. This observation is in direct support of the theoretical predictions of Guo et al. ${ }^{15}$

If we consider the $n B_{u}$ as the beginning of the free-electron continuum then we can assign its location in PPA-DBO, PPV-DOO, and PAT6 as 1.5, 1.6, and 1.4 times the ground state to first excited state transition energy, respectively. In all cases the $\mathrm{mA}_{9}$ state is located at approximately 1.3 times the ground state to first excited state transition energy, in good agreement with previous experimental results. $7-12,22$ In the case of PAT6, the $\mathrm{mA}_{g}$ state is located closer to the assigned continuum band than in PPA-DBO or PPV-DOO. This supports the results of calculations performed by Abe et al. that place the first $A_{g}$ character state very near the free-electron continuum. ${ }^{6}$ In contrast, the $\mathrm{mA}_{g}$ states of PPA-DBO and PPV-DOO are further from the assigned continuum band than the correlation perturbation to the SSH Hamiltonian would allow, suggesting that approach of Guo et al may be more appropriate. ${ }^{15}$

In considering the dispersion in nonlinear refraction near two-photon absorption peaks it becomes apparent that the behavior is opposite to that predicted by the Kramers-Kronig relationships or by the resonant denominator form of $\chi^{(3)}$ obtained from density matrix calculations. So far we have no explanation for this effect. It may be that we lack sufficient resolution to fully characterize the $n_{2}$ spectrum in this regime. It may also be related to the errors incurred when extrapolating $n_{2}$ in the presence of saturation, although this seems unlikely given the very definitive Z-scan signatures for positive or negative $n_{2}$. Since saturation is present, it may be an effect arising from a stark shift in the $A_{g}$ state position that results in frequent sign reversals of the resonant denominator when in the proximity of a two-photon resonance. Interestingly, the off-resonance $n_{2}$ is negative, in contrast to that observed in PTS single 
crystals and contrary to theoretical predictions. It seems that a more reasonable $n_{2}$ spectrum for all three polymers could be obtained by flipping the spectra about the energy axis.

Figure 5 summarizes the relative ordering and characteristics $\left(A_{g}\right.$ or $\left.B_{v}\right)$ of the obtained transitions, based on the experimental results. The width in energy of the boxes which identify the spectral peak locations indicates the error in determining the exact position of the spectral peaks.

\section{Conclusion}

Using the $Z$ scan technique we have resolved the most essential $A_{g}$ character state, the $\mathrm{mA}_{g}$, in PPA-DBO, PPV-DOO, and PAT6. In addition, the existence of the $\mathrm{mA}_{g}$ state above the $1 \mathrm{~B}_{u}$ exciton in all three polymers studied, clearly demonstrates that band theory alone is not sufficient to account for the excited states spectra of luminescent conducting polymers. These results also show the presence of other $A_{g}$ states besides the four essential states proposed in recent experiments and theories.8, 24 Furthermore, the close proximity of the $n B_{u}$ state to the higher energy $A_{g}$ states suggest the onset of the free-electron continuum, where even and odd-parity states exist coincidentally. The sensitivity of the $Z$ scan technique in directly measuring TPA and detecting dispersion in the nonlinear index of refraction near TPA peaks has also been demonstrated.

\section{Acknowledgments}

This work was supported in part by the Office of Naval Research, Grant No. N00014-94-1-0853, the DOE FG-03-96ER45490, and a grant from the University of Utah Research Committee.

\section{References}

1 S. Abe, Y. Shimoi, V. A. Shakin, et al., Mol. Cryst. Liq. Cryst. 256, 97-104 (1994).

2 P. C. M. McWilliams, G. W. Hayden, and Z. G. Soos, Phys. Rev. B 43, $9777-9791$

3 W. P. Su, J. R. Schrieffer, and A. J. Heeger, Phys. Rev. B 22, 2099-2111 (1980).

4 D. Guo, S. Mazumdar, S. N. Dixit, et al., Physical Review B 48, 1433-1459 (1993).

$5 \quad$ V. A. Shakin and S. Abe, Phys. Rev. B 50, 4306-4315 (1994).

6 S. Abe, M. Schreiber, W. P. Su, et al., Phys. Rev. B 45, 9432-9435 (1992).

C. J. Baker, O. M. Gelsen, and D. D. C. Bradley, Chemical Physics Letters 201, 127-131 (1993).

M. Cha, W. E. Torruellas, S. H. Yuan, et al., J. Opt. Soc. Am. B 12, 882-888 (1994).

S. A. Jeglinski, Z. V. Vardeny, Y. Ding, et al., Mol. Cryst. Liq. Cryst. 256, 87-96 (1994).

U. Lemmer, R. Fischer, J. Feldmann, et al., Chemical Physics Letters 203, 28-32 (1993).

J. M. Leng, S. Jeglinski, X. Wei, et al., Phys. Rev. Lett. 72, 156-159 (1994).

N. Pfeffer, P. Raimond, F. Charra, et al., Chemical Physics Letters 201, 357-363 (1993).

B. Lawrence, W. E. Torruellas, M. Cha, et al., Phys. Rev. Lett. 73, 597-600 (1994).

R. K. Meyer, R. E. Benner, Z. V. Vardeny, et al., in 1995 March Meeting of the American Physical Society, edited by B. H. Ripin (American Physical Society, San Jose, CA, 1995), Vol. 40, p. 103. D. Guo, S. Mazumdar, and S. N. Dixit, Synthetic Metals 49, 1-11 (1992).

16 D. Neher, A. Kaltbeitzel, A. Wolf, et al., in Conjugated Polymeric Materials: Opportunities in Electronics, Optoelectronics, and Molecular Electronics, edited by J. L. Bredas and R. R. Chance (Kluwer Academic Publishers, Amsterdam, 1990), p. 387-398.

17 K. S. Wong and Z. V. Vardeny, Synthetic Metals 49-60, 13-20 (1992).

18 W. H. Brown, Introduction to Organic Chemistry (Willard Grant Press, Boston, 1978).

M. Sheik-Bahae, A. A. Said, T. Wei. et al., IEEE J. of Quant. Electronics 26, 760-769 (1990).

Z. G. Soos and R. G. Kepler, Phys. Rev. B 43, 11908 (1991).

Z. G. Soos, S. Ramasesha, D. S. Galvao, et al., Synthetic Metals 64, 35-47 (1993).

A. Mathy, K. Ueberhofen, R. Schenk, et al., Phys. Rev. B 53, 4367-4376 (1996).

S. A. Hambir, G. J. Blanchard, and G. L. Baker, J. Chem. Phys. 102, 2295-2301 (1995).

D. Guo, in Theory of Optical Nonlinearity in p-Conjugated Polymers and Related Material (Univ. of Arizona, Tucson, 1993). 

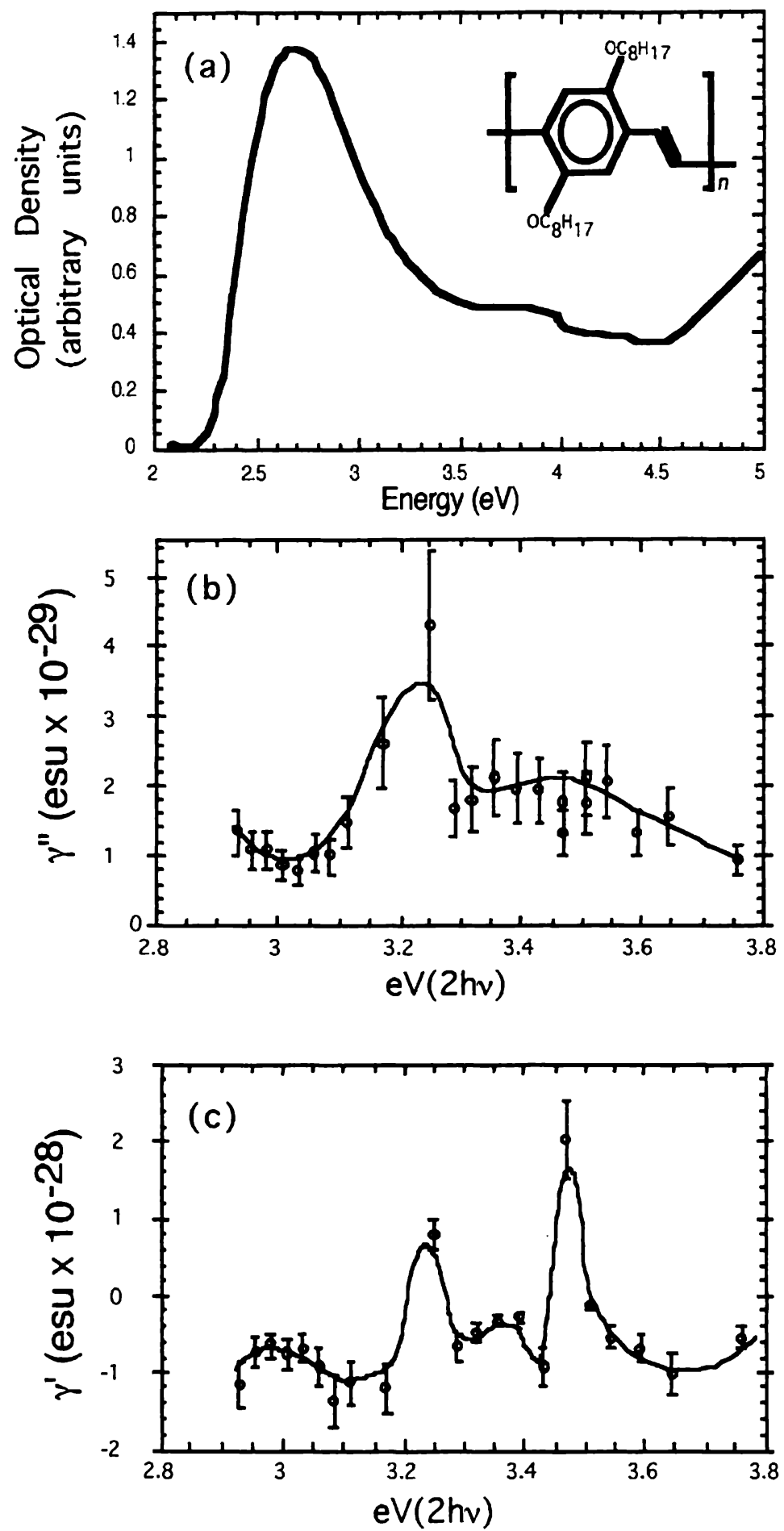

Figure 1: Linear and nonlinear spectra for PPV-DOO. Open circles depict actual data points and the solid lines are guides for the eye. a) Linear absorption spectra (Inset: chemical structure). Imaginary part of $\gamma$ (roughly proportional to TPA strength). c) Real part of $\gamma$, (rougly proportional to the nonlinear index of refraction, $\mathrm{n}_{2}$ ). 

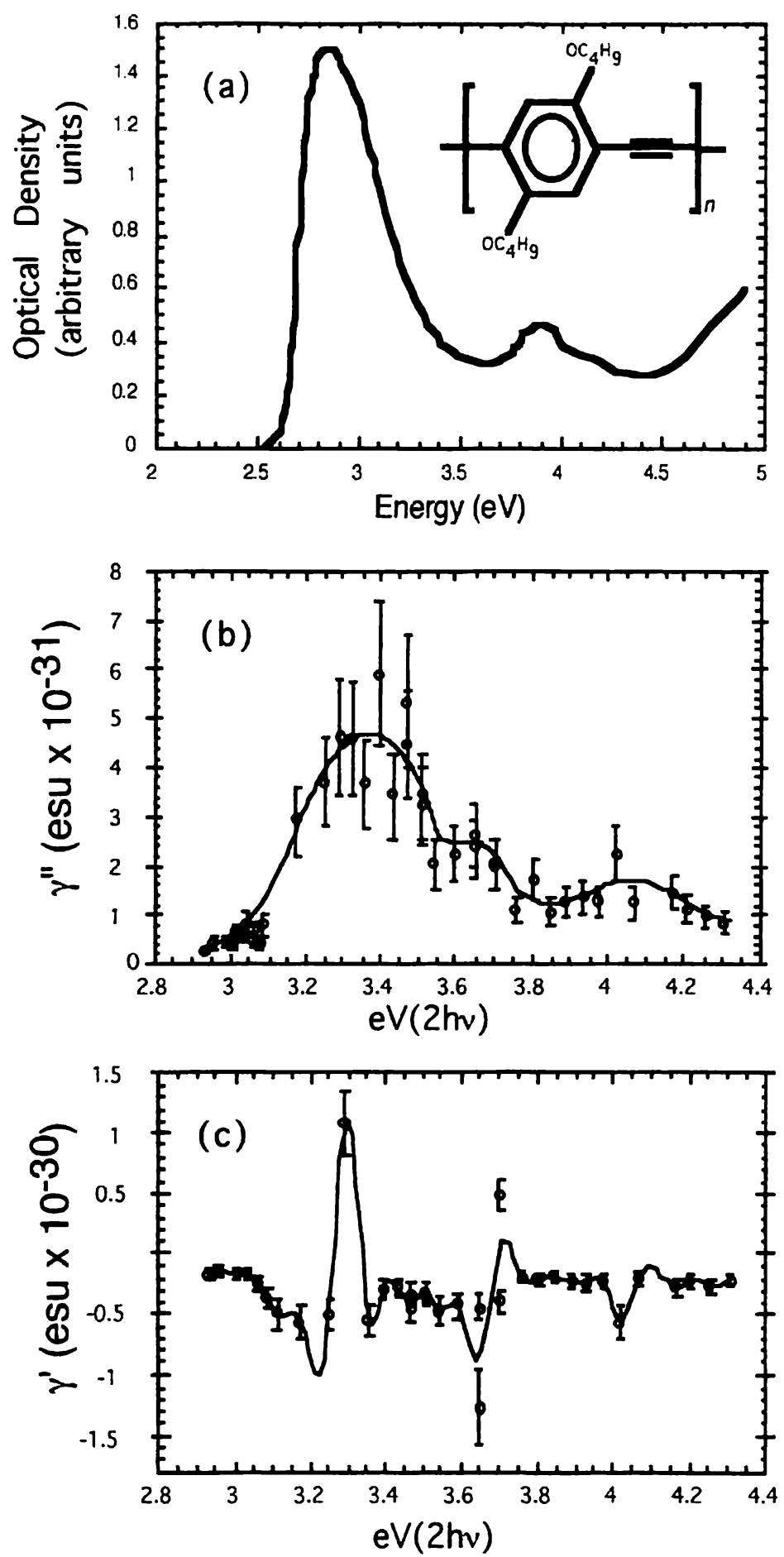

Figure 2: Linear and nonlinear spectra for PPA-DBO. Open circles depict actual data points and the solid lines are guides for the eye. a) Linear absorption spectra (Inset: chemical structure). b) Imaginary part of $\gamma$ (roughly proportional to TPA strength). c) Real part of $\gamma$, (rougly proportional to the nonlinear index of refraction, ' $\mathrm{n}_{2}$ ). 

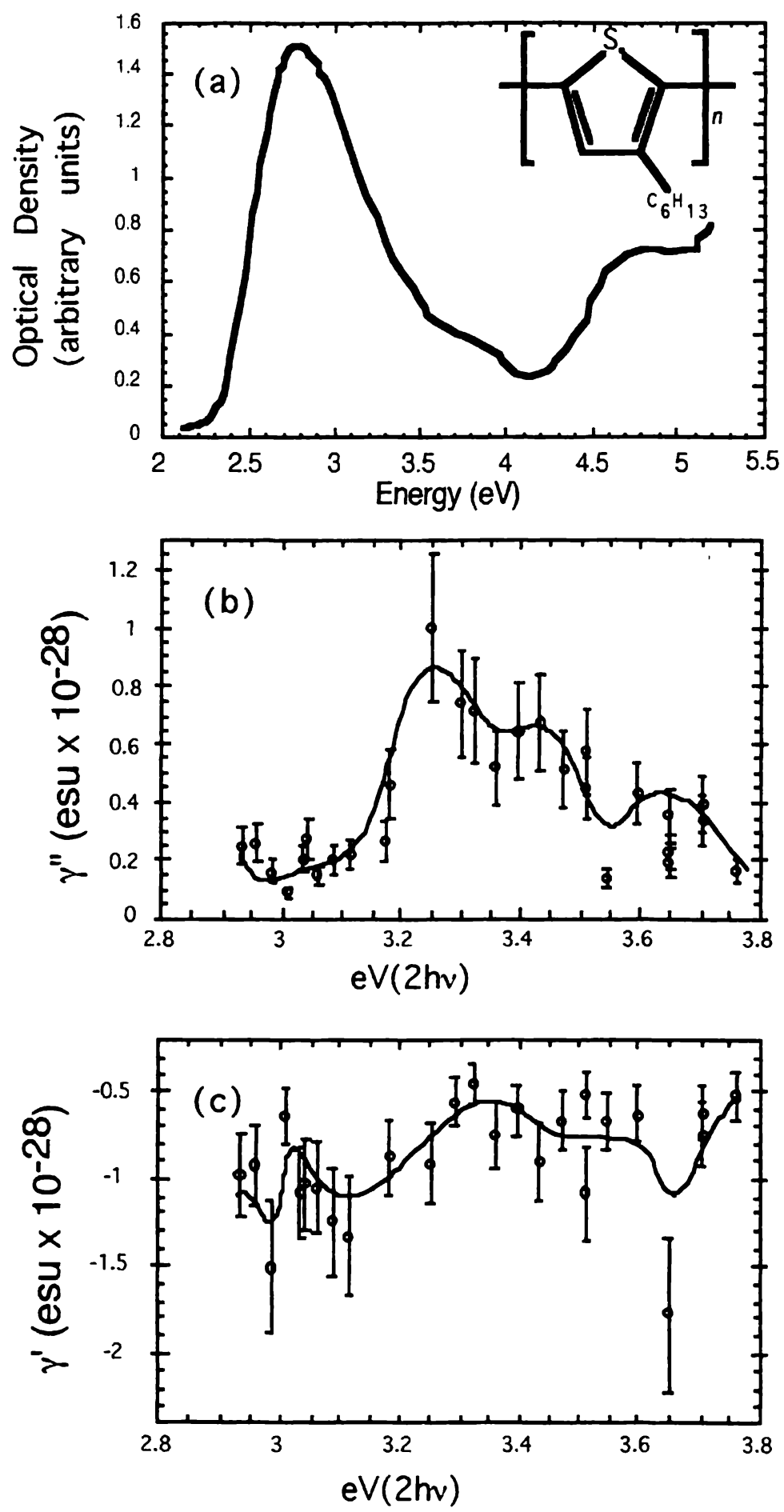

Figure 3: Linear and nonline ar spectra for PAT6. Open circles depict actual data points and the solid lines are guides for the eye. a) Linear absorption spectra (Inset: chemical structure). b) Imaginary part of $\gamma$ (roughly proportional to TPA strength). c) Real part of $\gamma$, (rougly proportional to the nonlinear index of refraction, $\mathrm{n}_{2}$ ). 


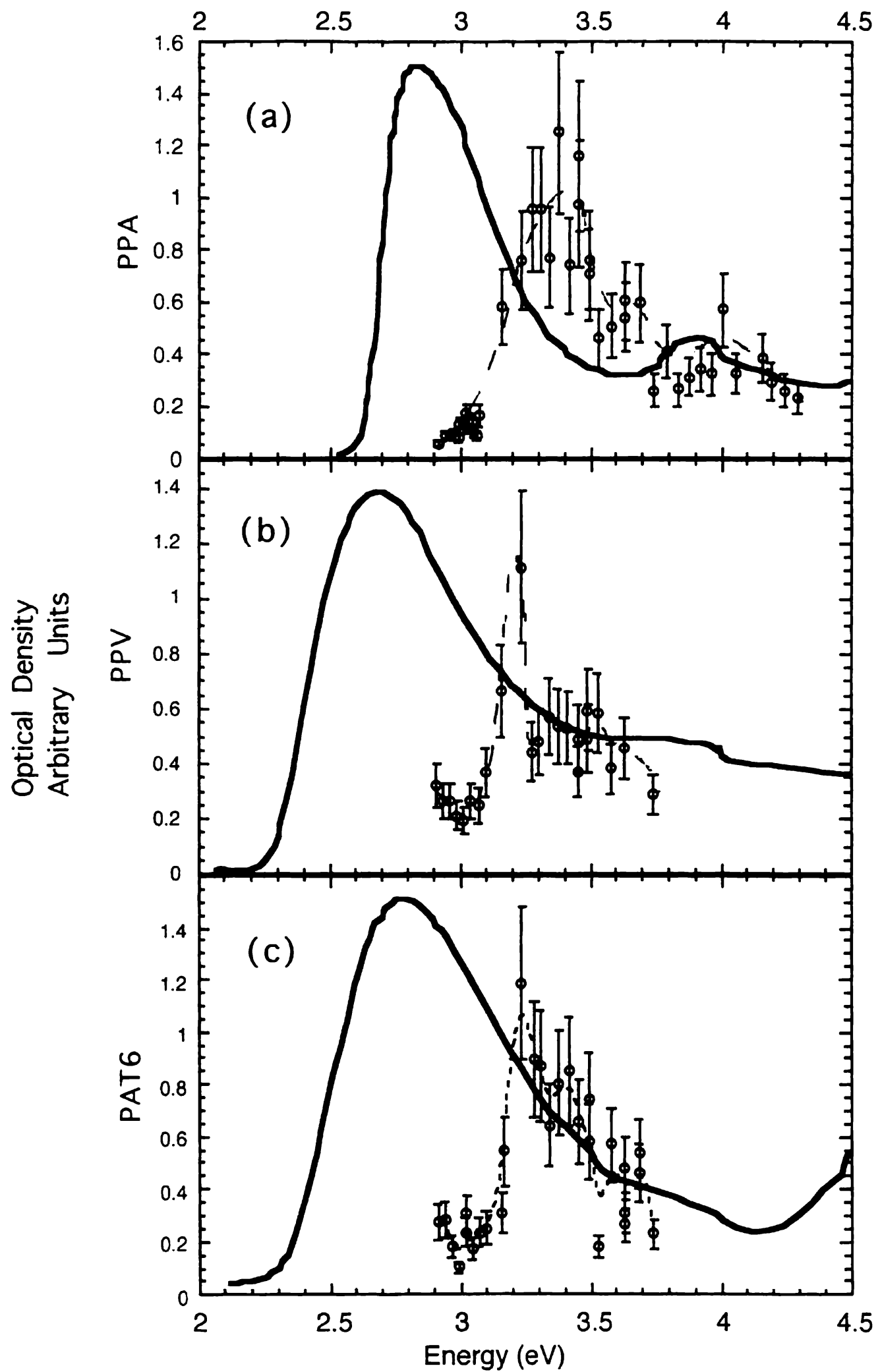

Figure 4: Linear and nonlinear absorption plotted coincidentally. a) PPA-DBO. b) PPV-DOO. c) PAT6. 


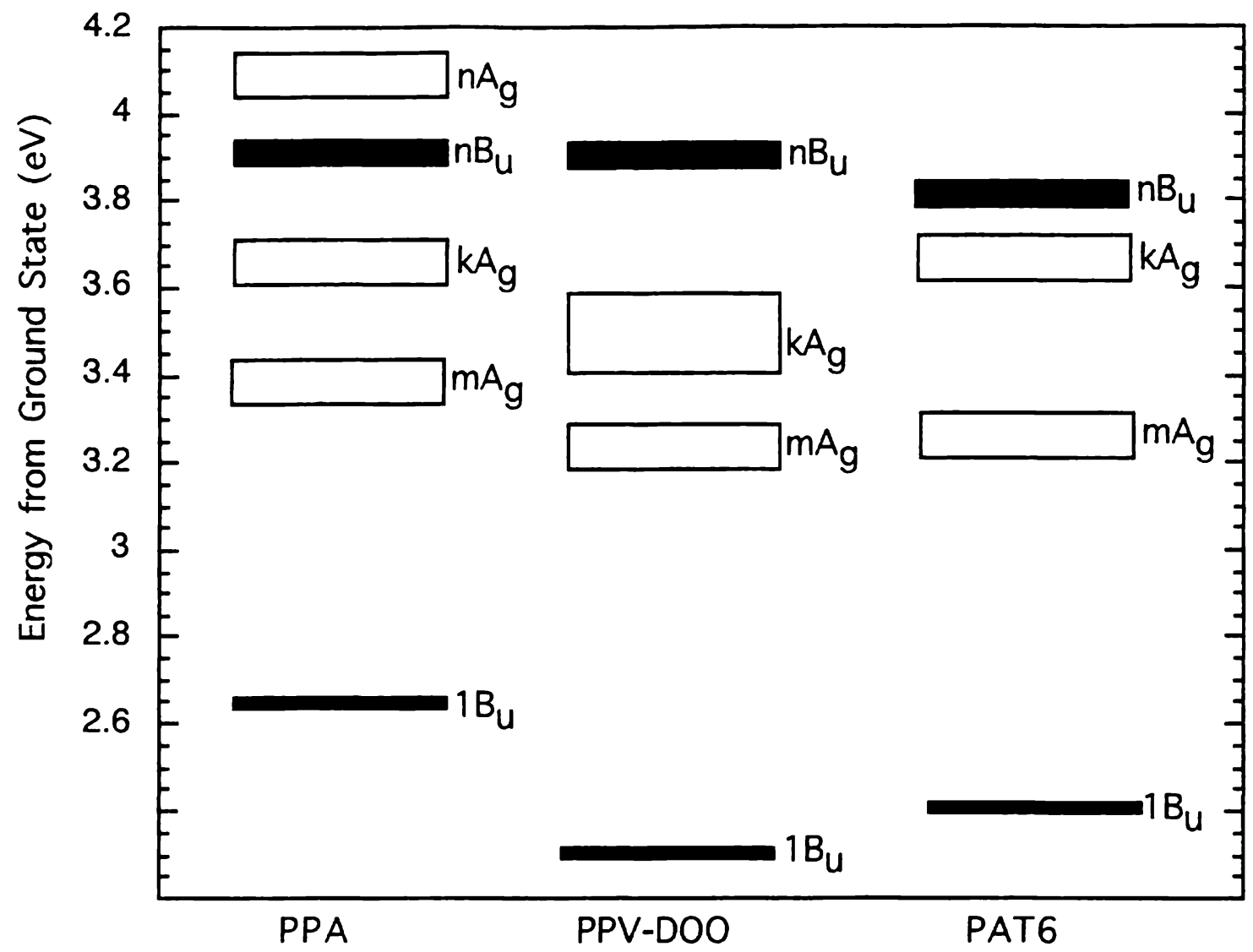

Figure 5: Energy level summary. Shaded blocks indicate $B_{u}$ character states while unshaded boxes indicate $A_{g}$ character states. The energetic width of boxes indicate the degree of uncertainty in fixing their location based on the uncertainty in the linear and nonlinear absorption data. 